\title{
Use of sequence-based typing for investigation of a case of nosocomial legionellosis
}

\section{Correspondence \\ Peter A. G. Tilley \\ p.tilley@provlab.ab.ca}

Received 3 June 2006

Accepted 11 August 2006

\author{
Sallene Wong, ${ }^{1}$ Kanti Pabbaraju, ${ }^{1}$ Veronica F. Burk, ${ }^{1}$ \\ George C. Broukhanski, ${ }^{2}$ Julie Fox, ${ }^{1,3}$ Tom Louie, ${ }^{4}$ Manuel W. Mah, ${ }^{4}$ \\ Kathryn Bernard ${ }^{5}$ and Peter A. G. Tilley ${ }^{1,3}$ \\ ${ }^{1}$ Provincial Laboratory for Public Health (Microbiology), Calgary site, Calgary, Alberta, Canada \\ ${ }^{2}$ Ontario Ministry of Health and Long-Term Care, Toronto, Ontario, Canada \\ ${ }^{3}$ Department of Microbiology and Infectious Diseases, University of Calgary, Alberta, Canada \\ ${ }^{4}$ Infection Prevention and Control, Calgary Health Region, Calgary, Alberta, Canada \\ ${ }^{5}$ National Microbiology Laboratory, Winnipeg, Manitoba, Canada
}

\begin{abstract}
A fatal case of nosocomial legionellosis in a low prevalence region (Calgary, Alberta, Canada) prompted investigation into the source of infection. Hospital water systems contaminated with Legionella pneumophila have been shown to pose a risk to compromised patients. Typing of an $L$. pneumophila serogroup 1 strain isolated from the patient using sequence-based typing (SBT) and amplified fragment length polymorphism (AFLP) analysis linked it to a persistent and widespread strain isolated from the hospital water system establishing a nosocomial mode of acquisition. Different SBT and AFLP patterns were determined for non-epidemiologically linked cases and isolates from different hospitals.
\end{abstract}

\section{INTRODUCTION}

Legionella pneumophila infection has been associated with fountains and respiratory therapy equipment suggesting that transmission occurs via inhalation (Woo et al., 1992). Microaspiration of contaminated potable water is another mode of transmission (Blatt et al., 1993; Stout \& Yu, 1997; $\mathrm{Yu}, 1993)$. The incidence of legionellosis in Alberta (Canada) is low compared to the eastern provinces of Ontario and Quebec, with 0.047 human cases per 100000 per year (Public Health Branch, Alberta Health and Wellness), and thus a case of nosocomial pneumonia due to L. pneumophila serogroup 1 that contributed to the death of a patient in an acute-care hospital in Calgary, Alberta, raised concern and prompted the analysis of water supplies.

Due to the ubiquitous presence of legionellae in nature, discriminatory molecular methods are required for investigation of clinical and environmental strains to determine the source of infection. Recently sequence-based typing (SBT) has been described as a simple, rapid, discriminatory and portable method for typing L. pneumophila strains (Gaia et al., 2003, 2005). Based on the sequence of six target genes, strains are assigned an SBT pattern number by the

Abbreviations: AFLP, amplified fragment length polymorphism; ER, emergency room; EWGLI, European Working Group for Legionella Infections; ICU, intensive care unit; SBT, sequence-based typing.

The GenBank accession numbers for the novel alleles of mompS and proA are D0309456 and D0309457, respectively.
European Working Group for Legionella Infections (EWGLI: www.ewgli.org). In this report, we used SBT to study the link between a rare nosocomial case of $L$. pneumophila infection and environmental isolates. We also performed amplified fragment length polymorphism (AFLP) analysis (Valsangiacomo et al., 1995) for confirmation of the SBT types.

\section{METHODS}

Isolation of L. pneumophila. L. pneumophila was isolated from bronchoalveolar lavage specimens on buffered charcoal yeast extract medium and from potable water by standard methods (Greenberg et al., 1998). Isolates from two other non-epidemiologically related community-acquired cases (C03B09917 and C04B12826) and $L$. pneumophila strain ATCC 33153 were included as controls. Colonies with Legionella morphology were identified using routine clinical laboratory methods and tested by the MONOFLUO Legionella pneumophila IFA test kit (Bio-Rad). Identification was confirmed by cellular fatty acid analysis, using the MIDI system with Sherlock software version 4.5 , and by $16 \mathrm{~S}$ ribosomal sequencing at the National Microbiology Laboratory, Winnipeg, Canada. Isolates were identified as serogroup 1 using monoclonal antibodies (ProLab).

SBT of $\boldsymbol{L}$. pneumophila. Genomic DNA was extracted from study isolates using the QIAamp DNA Mini kit (Qiagen). Six genes, flaA, pile, asd, mip, mompS and proA, were amplified using the primers and amplification protocol provided by the EWGLI. Amplified products were sequenced in both directions using the ABI PRISM BigDye Terminator v3.1 Cycle Sequencing kit in the ABI PRISM 3100-Avant Genetic Analyser (Applied Biosystems). Data collection used the Applied Biosystems Software v2.0. The sequences were 
edited and analysed using BioEdit v7.1.1 (http://www.mbio. ncsu.edu/BioEdit/bioedit.html). SBT allele numbers were assigned to the strains based on the EWGLI database.

AFLP. For the AFLP analysis, DNA was extracted using the TaKaRa DEXPAT method (TAKARA Bio Inc), and PCR, restriction digests and agarose gel electrophoresis were carried out according to methods described previously (Fry et al., 1999; Valsangiacomo et al., 1995).

\section{RESULTS AND DISCUSSION}

\section{Patient and environmental isolates}

A 74-year-old woman was admitted to hospital 1 due to a myocardial infarction. She was intubated in the emergency room (ER) and transferred to the intensive care unit (ICU), where she was stabilized. On day 10 she developed pneumonia, and L. pneumophila serogroup 1 was isolated from a bronchoalveolar lavage specimen. The infection was defined as nosocomial, based on the onset and the incubation period. She died on day 24 of cardiac complications. L. pneumophila serogroup 1 was isolated from potable water in the ER and ICU in the hospital, as well as from water samples from other hospitals in the city, but not from the residence of the index patient.

\section{Typing of Legionella environmental and clinical isolates}

The SBT allele numbers based on the six target genes are summarized in Table 1. The new alleles from this study have been submitted to the EWGLI SBT database and the National Center for Biotechnology Information database (GenBank accession numbers are: C04B12826 mompS, DQ309456; and C04B12826 proA, DQ309457). AFLP patterns of the control and samples are shown in Fig. 1.

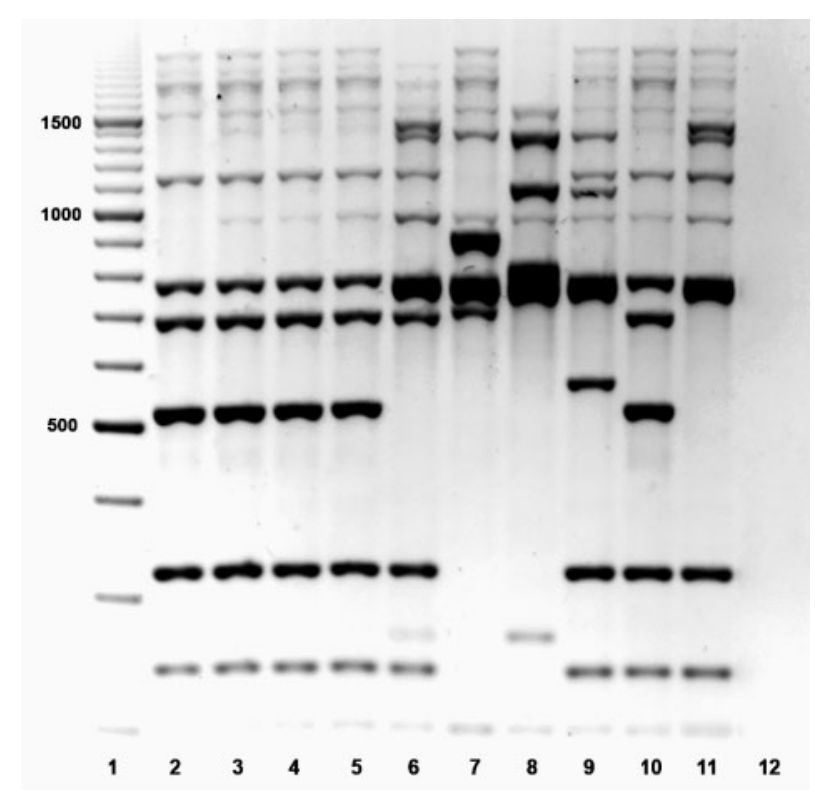

Fig. 1. AFLP patterns for L. pneumophila serogroup 1 patient and environmental isolates. Lanes: 1, $100 \mathrm{bp}$ ladder; 2, patient 1, hospital 1; 3, ICU sink 1, hospital 1; 4, ICU sink 2, hospital 1 ; 5 , ER sink 1, hospital 1; 6, ATCC 33153; 7, patient 2, hospital 3; 8, patient 3, hospital 3; 9, ICU sink 1, hospital 3; 10, ICU sink 1, hospital 2; 11, ICU sink 1, hospital 4; 12, Negative PCR control.

The alphabetical designations based on the electrophoresis pattern are included in Table 1.

SBT and AFLP of the samples (Table 1) revealed that the isolate from the index (deceased) patient (C03B16848, hospital 1) had the same sequence type profile across the six target genes $(1,4,3,1,1,1)$ as isolates recovered from water in

Table 1. Summary of SBT and AFLP patterns of $L$. pneumophila serogroup 1 patient and environmental isolates

\begin{tabular}{|c|c|c|c|c|}
\hline $\begin{array}{l}\text { Sample } \\
\text { identification }\end{array}$ & $\begin{array}{c}\text { Type of } \\
\text { specimen }^{*}\end{array}$ & $\begin{array}{l}\text { Isolation origin, hospital, } \\
\text { date of collection }\end{array}$ & $\begin{array}{c}\text { SBT allele numbers } \\
(\text { flaA, pilE, asd, mip, mompS, proA })\end{array}$ & AFLP pattern type \\
\hline C03B16848 & BAL & Patient 1, hospital 1, 27 November 2003 & $(1,4,3,1,1,1)$ & A \\
\hline C03B17881 & Water & ICU sink 2, hospital 1, 17 December 2003 & $(1,4,3,1,1,1)$ & A \\
\hline C04B04689 & Water & ICU sink 2, hospital 1, 23 March 2004 & $(1,4,3,1,1,1)$ & ND \\
\hline C04B07897 & Water & ICU sink 1, hospital 2, 1 June 2004 & $(1,4,3,1,1,1)$ & A \\
\hline C03B09917 & BAL & Patient 2, hospital 3, 15 July 2003 & $(3,4,1,1,14,9)$ & $\mathrm{B}$ \\
\hline C04B12826 & BAL & Patient 3, hospital 3, 9 September 2004 & $(12,9,2,5,27,20)$ & E \\
\hline C04B11181 & Water & ICU sink 1, hospital 3, 13 August 2004 & $(6,10,15,28,21,3)$ & $\mathrm{C}$ \\
\hline C04B11757 & Water & ICU sink 1, hospital 4, 26 August 2004 & $(7,6,17,3,11,11)$ & $\mathrm{D}$ \\
\hline
\end{tabular}

ND, Not determined.

${ }^{\star}$ BAL, Bronchoalveolar lavage. 
nearby ICU sinks (C03B17576 and C03B17881, hospital 1). Further sampling of hospital sinks outside of the ICU in hospital 1 ( $n=35$ samples) showed that $14 \%$ of water samples harboured $L$. pneumophila including that from the ER sink where the patient was admitted (C04B05011, hospital 1), and these isolates had the same SBT type as the patient. Water from the index patient's residence was negative for Legionella consistent with the nosocomial acquisition of infection. Potable water supplies in hospital wards housing highly compromised patients in three additional hospitals were tested for L. pneumophila. Water obtained from a nearby hospital (C03B07897, hospital 2) that is supplied from the same water reservoir as hospital 1 contained $L$. pneumophila with the same sequence profile, $1,4,3,1,1,1$. L. pneumophila isolated from water from two more distant hospitals in the same city (C04B11181, hospital 3; and C04B11757, hospital 4) had different L. pneumophila SBT types. These hospitals derive their water from a different water reservoir, and these isolates were included as non-epidemiologically related controls.

The two non-epidemiologically linked communityacquired cases (C03B09917 and C04B12826 from hospital 3 ) had different SBT types and AFLP patterns compared to the isolates from the index patient and from water of the various hospitals, consistent with their communityacquired source of infection.

The water system of the affected hospital was superheated (greater than $71{ }^{\circ} \mathrm{C}$ for more than $30 \mathrm{~min}$ ) and flushed as this approach could be instituted quickly to suppress $L$. pneumophila from the water supply to the affected wards. Despite this treatment, it was still possible to isolate $L$. pneumophila with the same sequence profile after 3 months (C04B04689 and C04B05011, hospital 1). A copper-silver ionizer was installed to control Legionella in the water system, but L. pneumophila of the same SBT type persisted in the water (C05B03153, hospital 1).

Although this is a small study, the finding of identical SBT types in the patient and hospital water sources in units occupied by the patient strongly implicates the hospital potable water system as the source of the nosocomial infection. L. pneumophila SBT type $1,4,3,1,1,1$ has been shown to be a widespread environmental strain, isolated in Japan (Amemura-Maekawa et al., 2005) and Europe (Scaturro et al., 2005), thus calling into question the discriminatory power of SBT for this strain. In this study, however, we were able to establish an epidemiological link between the patient and water isolates and show that this strain was not seen in other patients or in non-epidemiologically linked water samples in this region. The results were also confirmed by AFLP, a highly discriminatory method for Legionella typing (Fry et al., 2000).

This study extends recent reports (Amemura-Maekawa et al., 2005; Scaturro et al., 2005; Perola et al., 2005) by demonstrating that SBT using the EWGLI database for $L$. pneumophila allele designations is useful for investigation of nosocomial legionellosis cases and hospital environmental strains. As the clinical and epidemiological interpretation of environmental isolates is controversial, a typing method with this level of discriminatory power has great potential for assisting in epidemiological studies. Until the epidemiology of environmental strains is better characterized, however, the discriminatory power of SBT can be increased by using monoclonal antibody serogrouping (Gaia et al., 2005) and confirmatory methods such as AFLP or PFGE (Amemura-Maekawa et al., 2005).

\section{ACKNOWLEDGEMENTS}

We thank the technologists at the Provincial Laboratory for Public Health (Bacteriology), Calgary, and L. De Winter at the National Microbiology Laboratory, Winnipeg, for their technical assistance. The funding for this study was provided by the Calgary Health Region and Alberta Health and Wellness (Alberta, Canada).

\section{REFERENCES}

Amemura-Maekawa, J., Kura, F., Chang, B. \& Watanabe, H. (2005). Legionella pneumophila serogroup 1 isolates from cooling towers in Japan form a distinct genetic cluster. Microbiol Immunol 49, 1027-1033.

Blatt, S. P., Parkinson, M. D., Pace, E., Hoffman, P., Dolan, D., Lauderdale, P., Zajac, R. A. \& Melcher, G. P. (1993). Nosocomial Legionnaires' disease: aspiration as a primary mode of disease acquisition. Am J Med 95, 16-22.

Fry, N. K., Exiou-Daniel, S., Bangsborg, J. M. \& 11 other authors (1999). A multicenter evaluation of genotypic methods for the epidemiologic typing of Legionella pneumophila serogroup 1: results of a pan-European study. Clin Microbiol Infect 5, 462-477.

Fry, N. K., Bangsborg, J. M., Bernander, S. \& 11 other authors (2000). Assessment of intercentre reproducibility and epidemiological concordance of Legionella pneumophila serogroup 1 genotyping by amplified fragment length polymorphism analysis. Eur J Clin Microbiol Infect Dis 19, 773-780.

Gaia, V., Fry, N. K., Harrison, T. G. \& Peduzzi, R. (2003). Sequencebased typing of Legionella pneumophila serogroup 1 offers the potential for true portability in legionellosis outbreak investigation. J Clin Microbiol 41, 2932-2939.

Gaia, V., Fry, N. K., Afshar, B., Luck, P. C., Meugnier, H., Etienne, J., Peduzzi, R. \& Harrison, T. G. (2005). Consensus sequence-based scheme for epidemiological typing of clinical and environmental isolates of Legionella pneumophila. J Clin Microbiol 43, 2047-2052.

Greenberg, A. E., Clesceri, L. S. \& Eaton, A. D. (editors) (1998). Legionella. In Standard Methods for the Examination of Water and Wastewater, pp. 9-106-9-109. Washington: American Public Health Association, American Water Works Association, Water Environment Federation.

Perola, O., Kauppinen, J., Kusnetsov, J., Karkkainen, U. M., Luck, P. C. \& Katila, M. L. (2005). Persistent Legionella pneumophila colonization of a hospital water supply: efficacy of control methods and a molecular epidemiological analysis. APMIS 113, 45-53.

Scaturro, M., Losardo, M., De Ponte, G. \& Ricci, M. L. (2005). Comparison of three molecular methods used for subtyping of Legionella pneumophila strains isolated during an epidemic of legionellosis in Rome. J Clin Microbiol 43, 5348-5350.

Stout, J. E. \& Yu, V. L. (1997). Legionellosis. N Engl J Med 337, 682-687. 
Valsangiacomo, C., Baggi, F., Gaia, V., Balmelli, T., Peduzzi, R. \& Piffaretti, J. C. (1995). Use of amplified fragment length polymorphism in molecular typing of Legionella pneumophila and application to epidemiological studies. J Clin Microbiol 33, $1716-1719$.
Woo, A. H., Goetz, A. \& Yu, V. L. (1992). Transmission of legionella by respiratory equipment and aerosol generating devices. Chest 102, $1586-1590$.

Yu, V. L. (1993). Could aspiration be the major mode of transmission for legionella? Am J Med 95, 13-15. 